\title{
Recurrence of adenoma after curative endoscopic submucosal dissection for a rectal intramucosal adenocarcinoma in adenoma
}

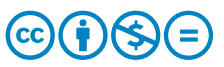

\author{
Authors \\ Yoshinori Morita', Eiji Umegaki ${ }^{1}$, Yuzo Kodama' \\ Institutions \\ 1 Division of Gastroenterology, Department of Internal \\ Medicine, Kobe University Graduate School of Medicine, \\ Kobe, Japan \\ 2 Department of Endoscopy, Kobe University Hospital, \\ Kobe, Japan \\ 3 Department of Gastroenterology, Kishiwada Tokushukai \\ Hospital, Kishiwada, Japan \\ 4 Department of Pathology, Kishiwada Tokushukai \\ Hospital, Kishiwada, Japan \\ 5 Taniguchi Gastrointestinal Endoscopy Clinic, Yao, Japan \\ 6 Department of Gastroenterology, Fuchu Hospital, Izumi, \\ Japan
}

Yoshiko Nakano, Takashi Toyonaga ${ }^{2,3}$, Eisei Nishino ${ }^{4}$, Taro Inoue ${ }^{3}$, Isato Shinjo ${ }^{5}$, Hajime Han-no ${ }^{6}$, Shinwa Tanaka ${ }^{1}$,

submitted 4.12.2018

accepted after revision 25.1.2019

Bibliography

DOI https://doi.org/10.1055/a-0867-9737 |

Endoscopy International Open 2019; 07: E621-E624

(c) Georg Thieme Verlag KG Stuttgart · New York

ISSN 2364-3722

Corresponding author

Takashi Toyonaga, MD, PhD, Department of Endoscopy, Kobe University Hospital, 7-5-1 Kusunoki-cho, Chuo-ku,
Kobe, Japan

Fax: +81-78-382-6309

toyonaga@med.kobe-u.ac.jp

\section{ABSTRACT}

Background and study aims A 71-year-old female underwent endoscopic submucosal dissection (ESD) for a subcircumferential lateral-spreading rectal tumor. Pathological examination showed an intramucosal adenocarcinoma in villous adenoma (size: $155 \times 140 \mathrm{~mm}$ ), which had been curatively resected with negative margins. However, follow-up colonoscopy revealed a tumor at the ulcer scar site, which soon grew into a circumferential lesion. Nineteen months after the first ESD procedure, additional ESD was performed for the recurrent lesion, which was resected en bloc without any adverse events, although severe fibrosis was noted in the submucosa. Pathological examination revealed a villous adenoma similar to the primary lesion with negative margins, but tumor cell nests were also present in the submucosa, which implied that tumor cell implantation had occurred during the first ESD. The post-ESD ulcer bed was subjected to argon plasma coagulation to prevent tumor recurrence after confirmation of the pathological results. There have not been any signs of recurrence during 9 years of follow-up.

\section{Introduction}

Endoscopic submucosal dissection (ESD) has already become a standard treatment for superficial tumors in the gastrointestinal tract [1]. The recurrence rate for such tumors after curative resection is reported to be very low $[2,3]$. However, there have been a few reports about recurrence of such tumors after curative resection, which was considered to have occurred due to tumor cell implantation ( $\mathrm{TCl}$ ) during ESD [4-6]. These cases included two cases of local recurrence in the rectum after ESD, and a case in which it was suspected that esophageal squamous carcinoma cells had implanted into a post-ESD gastric ulcer. The first two cases were treated via surgical resection, and the last case was managed with best supportive care because local recurrence of esophageal cancer was also detected.

On the other hand, we experienced a case in which adenoma recurred and exhibited rapid circumferential growth in the rectum after curative ESD resection, which was considered to have been caused by $\mathrm{TCl}$ during ESD. The recurrent lesion was completely resected via another ESD.

We present this case to alert clinicians to risk of $\mathrm{TCl}$ during ESD and the fact that even adenomas can grow rapidly, as well as to show that it is possible to resect recurrent circumferential lesions using ESD. 

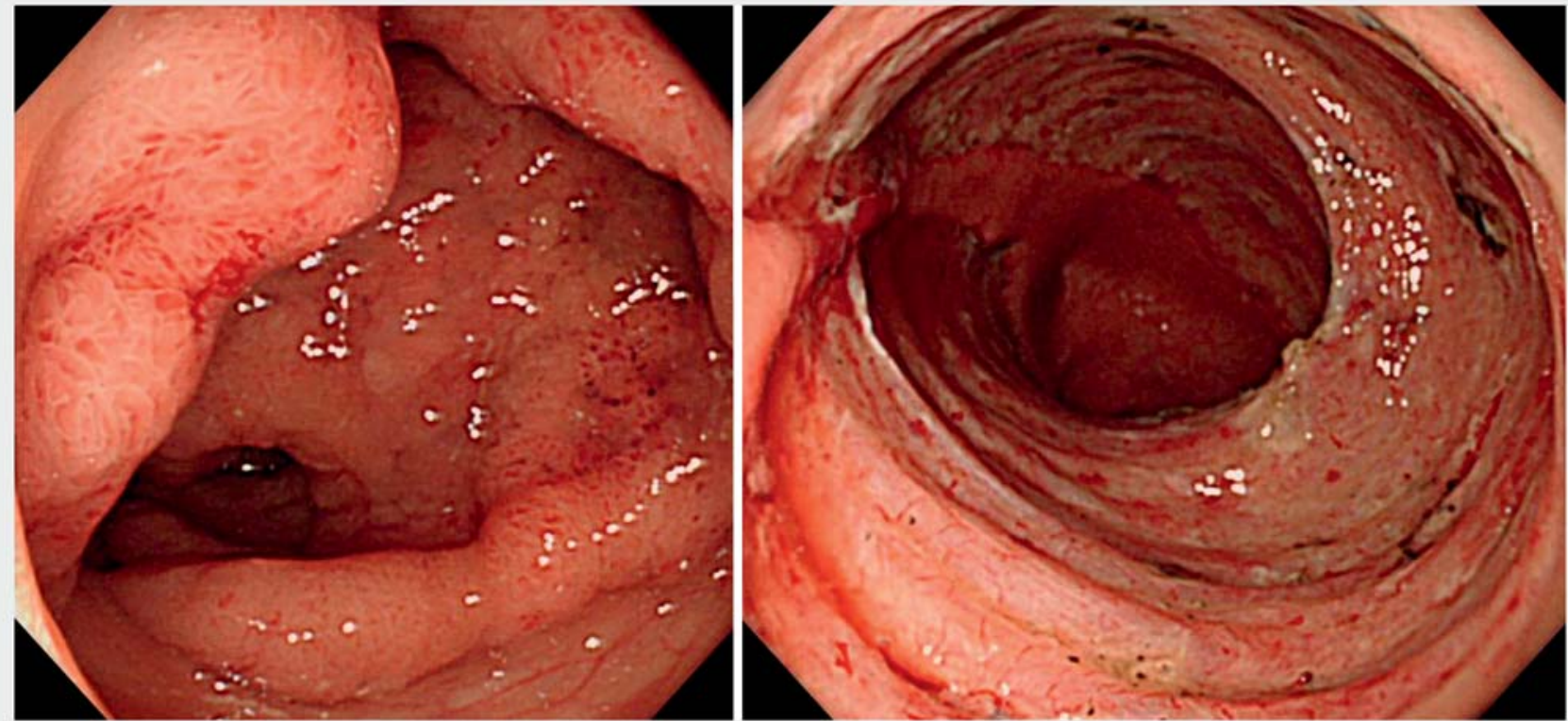

Fig. 1 Subcircumferential lateral-spreading tumor in the rectum was resected by endoscopic submucosal dissection.
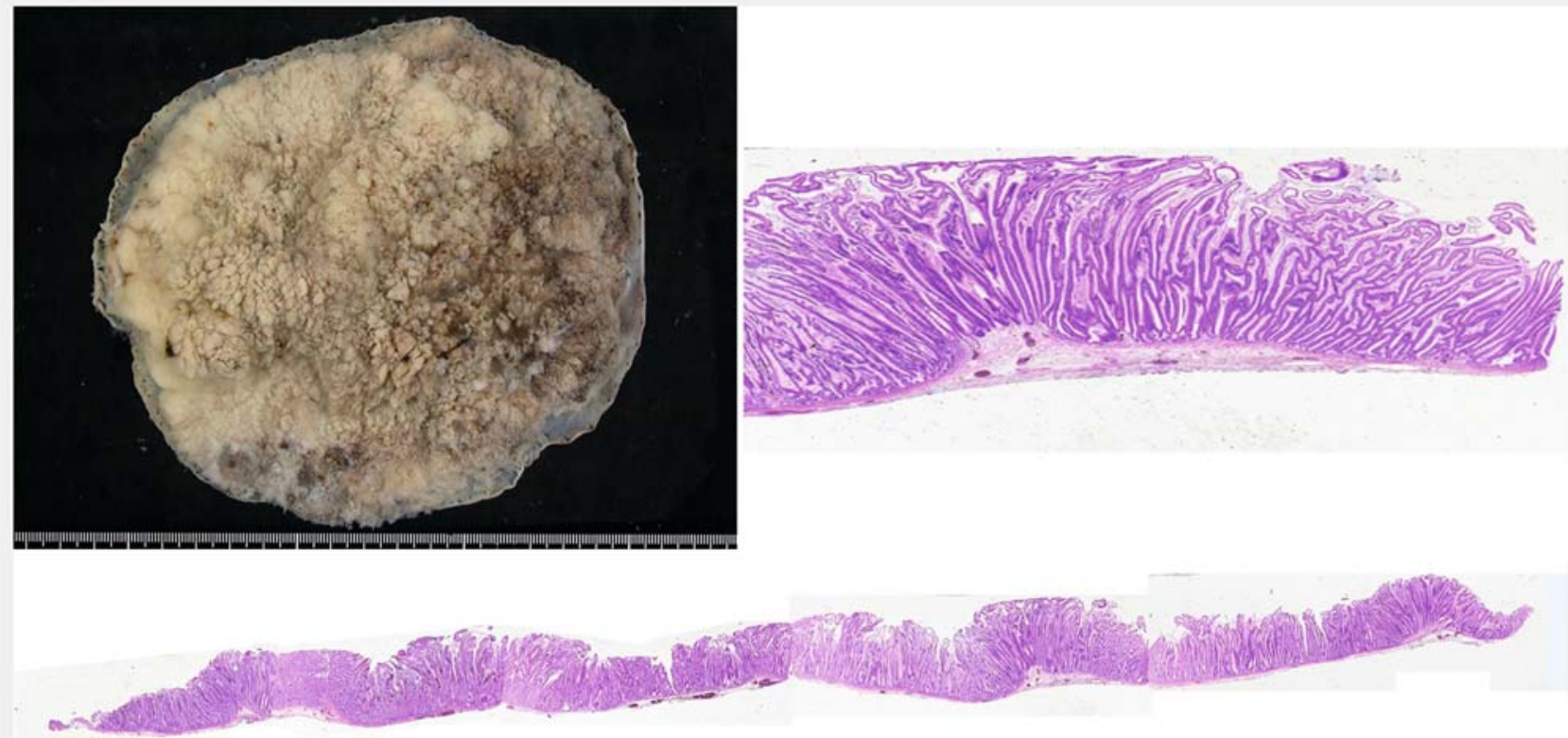

Fig. 2 The resected specimen. Pathological examination showed an intramucosal adenocarcinoma in villous adenoma (size: $155 \times 140 \mathrm{~mm})$, which had been curatively resected with negative margins.

\section{Case report}

A 71-year-old female with a medical history of hypertension was diagnosed with a subcircumferential lateral-spreading rectal tumor. Magnified endoscopy led to a suspicion of intramucosal cancer in adenoma, and ESD was indicated. ESD was performed using a Flush knife (Fujifilm Medical Co., Ltd., Tokyo, Ja- pan), without any adverse events (AEs) ( $\triangleright$ Fig. 1$)$. Grossly, the tumor measured $155 \times 140 \mathrm{~mm}$, and its lateral margins were negative. Microscopic examination revealed an intramucosal adenocarcinoma in villous adenoma, which had been curatively resected and did not exhibit lymphovascular invasion ( $>$ Fig. 2). A rectal stricture occurred after ESD, and it was treated using a bougie with a short-caliber tip with a transparent hood (ST 

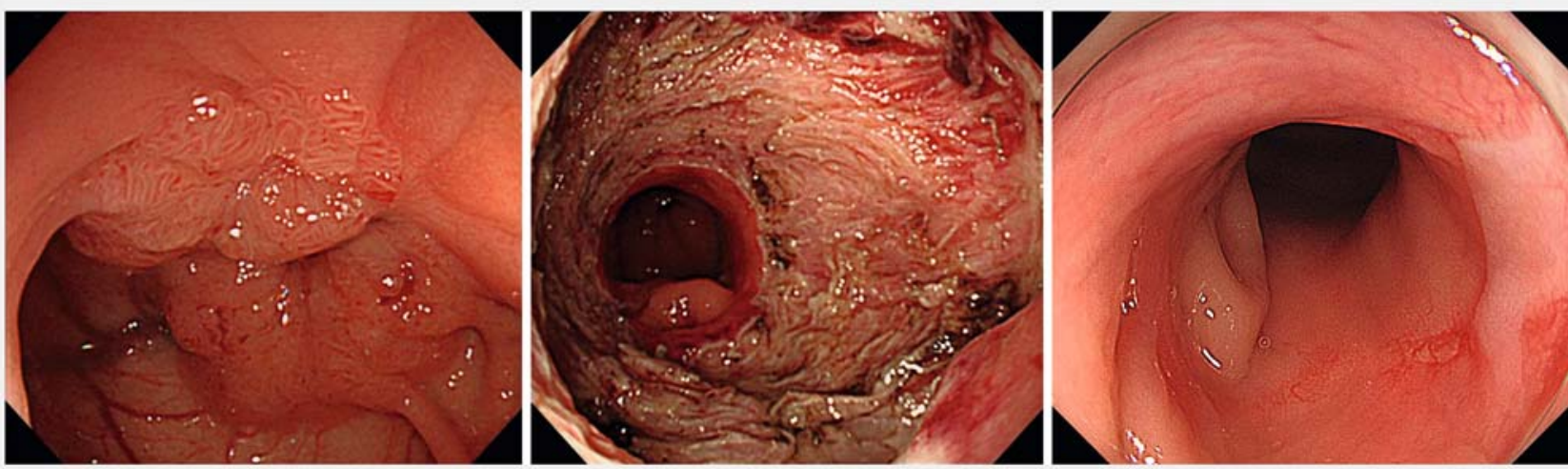

Fig. 3 A tumor was found at the ulcer scar site during follow-up colonoscopy and soon grew into a circumferential lesion. ESD was performed for the recurrent lesion, which was resected en bloc without adverse events. Follow-up colonoscopy performed at 9 years postsurgery showed no tumor recurrence or stricture.

hood). However, 2 1/2 months after ESD, an elevated lesion was found at the ulcer scar site during the course of dilation therapy. A biopsy specimen showed an adenoma, and recurrence was suspected. In spite of this, it was considered that post-ESD progression of fibrosis was most marked during this period and that it would take about 6 months to alleviate the fibrosis. Furthermore, the patient did not want to undergo frequent endoscopic procedures and so was observed.

At follow-up colonoscopy performed 15 months later, the tumor was found to have grown into a circumferential lesion ( $\triangleright$ Fig.3). With the patient's agreement, ESD was performed for the recurrent lesion using a Flush knife. Extremely severe fibrosis was seen, and the submucosal layer had almost completely disappeared. Therefore, we partially dissected at the level of the muscle layer to completely resect the tumor. The tumor was resected en bloc without any AEs. Pathological examination revealed a villous adenoma similar to the first ESD specimen with a specific feature such as pointed and elongated papilla structure, with negative margins. Tumor cell nests also were present in the submucosa ( $\triangleright$ Fig. 4 ), which implied that $\mathrm{TCl}$ had occurred during the first ESD procedure. Furthermore, staining for Ki67 tissue expression was performed using the anti-Ki67 antibody for the primary and additional ESD specimens, which showed the percentages of positively stained tumor cells were $16.6 \%$ and $32.8 \%$ in the adenomatous area of the primary lesion and recurrent lesion respectively.

To prevent tumor recurrence, the post-ESD ulcer bed was subjected to argon plasma coagulation after confirmation of the pathological results. Although postoperative stricture was noted, it was resolved with six sessions of endoscopic balloon dilation.

There have not been any signs of recurrence or stricture during the 9-year follow-up period ( $\triangleright$ Fig. 3 ).
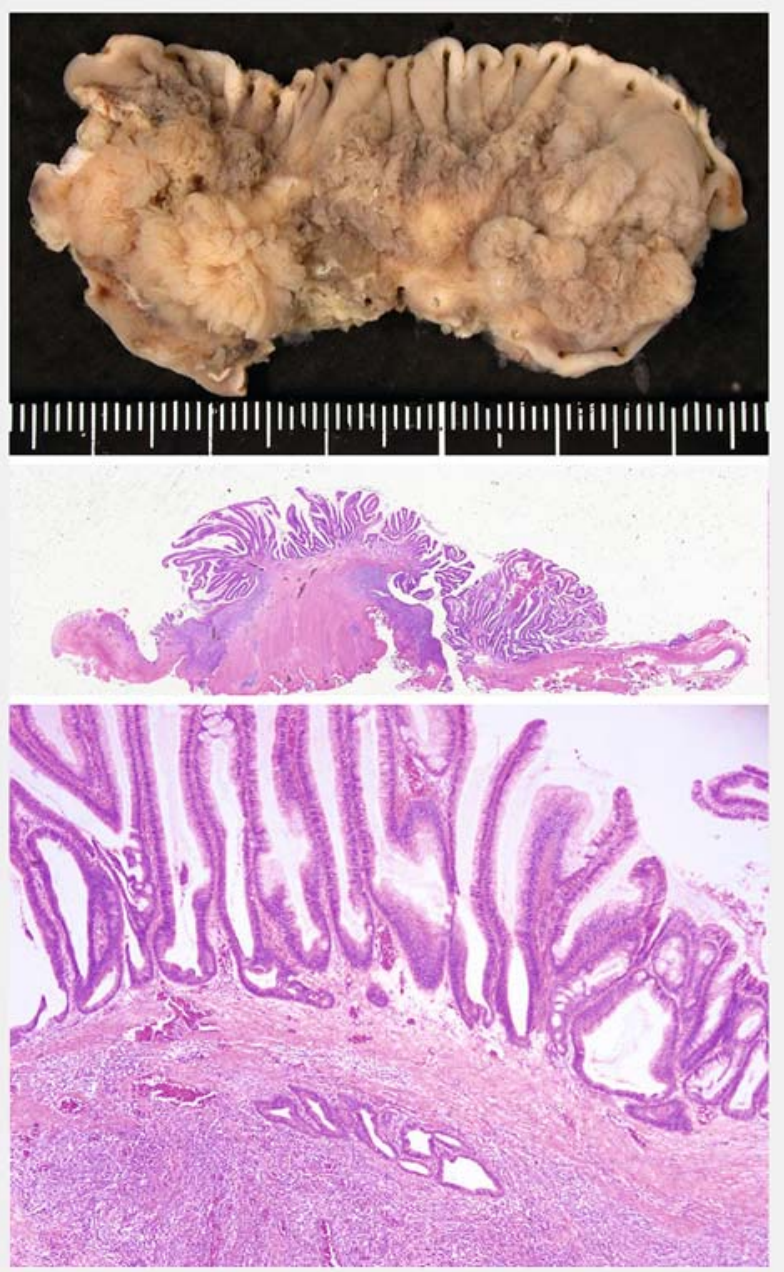

- Fig. 4 Pathological examination revealed a villous adenoma the same as the primary lesion with negative margins, but tumor cell nests were also present in the submucosa. 


\section{Discussion}

The current case showed that mucosal rectal adenomas can recur and exhibit rapid growth, producing circumferential lesions, after curative ESD resection. In the current case, the recurrent lesion was considered to have been caused by $\mathrm{TCl}$ during the ESD procedure, because the primary lesion was an intramucosal adenocarcinoma in adenoma and completely resected with negative lateral margins. There have been some case reports of cancer cell implantation in the rectum or stomach after curative ESD [4-6].

To the best of our knowledge, our case is the first reported case of recurrence of a rectal adenoma after ESD.

It has been reported that tumor cells are exfoliated into the intestinal lumen during ESD [7]. After submucosal dissection, the ulcer bed is exposed to these exfoliated tumor cells for a long time during ESD, which can lead to TCl.

Growth of an adenoma into a circumferential lesion within a year, as was seen in the current case, seems to be very rare, because adenomas usually grow slowly. In the previously mentioned reports on $\mathrm{TCl}$, recurrent tumors also grew rapidly, e. g., advanced cancer was diagnosed at the post-ESD ulcer site within a year of curative ESD for mucosal lesions [5, 6]. These reports suggest that recurrence due to $\mathrm{TCl}$ during endoscopic resection might be related to increased tumor enlargement and progression. Tanaka et al. reported that proliferative activity in recurrent tumors was significantly higher than that in primary tumors [8]. Also in the current case, the recurrent lesion showed higher Ki67 expression than the primary lesion although the morphologies and structures of tumor cells were similar, containing a specific feature. The increment of proliferative activity in the recurrent tumor presumably would also apply to $\mathrm{TCl}$ during ESD. Furthermore, post-ESD ulcers at sites where tumor vessels had gathered and tissue regeneration with inflammation that is prompted by ESD might represent fertile ground for $\mathrm{TCl}$.

In addition to risk of TCl during ESD, the possibility that the recurrent tumor will exhibit aggressive proliferation is threatening for patients.

Intraluminal lavage with $>1000 \mathrm{~mL}$ fluid after ESD reportedly is useful for removing exfoliated tumor cells after colorectal ESD [7]. Use of this method may contribute to preventing $\mathrm{TCI}$ after ESD.

Endoscopists who perform ESD for gastrointestinal tract tumors should be aware of the possibility of $\mathrm{TCl}$ and should take countermeasures to prevent it.

Furthermore, it should also be noted that the recurrent circumferential lesion seen in the current case was successfully treated via an additional ESD. Additional endoscopic treatment for recurrent tumors is associated with technical difficulties because the initial procedure usually causes fibrosis in the submucosal layer. We have previously reported that curative and effective en bloc resection can be achieved in such cases [9]. In this method, the non-fibrotic submucosa around the fibrotic region is first dissected, followed by careful dissection of the fibrotic area between the blood vessel network of the submucosa and the muscle layer until the initial dissection field has been reached. In the current case, fibrosis was extremely severe, and the submucosal layer had almost disappeared. Therefore, we partial dissected at the level of the muscle layer to ensure that complete tumor resection was achieved. This led to development of a new method, perianal endoscopic myectomy, for rectal lesions with severe fibrosis that exhibit the muscle-retracting sign, in which dissection is performed between the inner circular and outer longitudinal muscles [10], though it must be noted that this technique should be performed carefully, because its safety has not been fully confirmed.

Hence, ESD can be indicated for large recurrent lesions with severe fibrosis if they are considered to be superficial.

\section{Conclusion}

In conclusion, $\mathrm{TCl}$ can occur during ESD of adenomas, and the proliferative activity of the resultant recurrent tumors can be high.

\section{Competing interests}

Dr. Toyonaga invented the Flush Knife in conjunction with Fujifilm and receives royalties from its sale.

\section{References}

[1] Tanaka S, Oka S, Kaneko I et al. Endoscopic submucosal dissection for colorectal neoplasia: possibility of standardization. Gastrointest Endosc 2007; 66: 100-107

[2] Yamada M, Saito Y, Takamaru H et al. Long-term clinical outcomes of endoscopic submucosal dissection for colorectal neoplasms in 423 cases: a retrospective study. Endoscopy 2017; 49: $233-242$

[3] Shigita K, Oka S, Tanaka S et al. Long-term outcomes after endoscopic submucosal dissection for superficial colorectal tumors. Gastrointest Endosc 2017; 85: 546 - 553

[4] Inoue T, Fujii H, Koyama F et al. Local recurrence after rectal endoscopic submucosal dissection: a case of tumor cell implantation. Clin J Gastroenterol 2014; 7: 36-40

[5] Shinhata $\mathrm{H}$, Yamamoto $\mathrm{H}$, Sunada $\mathrm{K}$ et al. Advanced rectal carcinoma caused by tumor cell implantation after curative endoscopic submucosal dissection of an intramucosal rectal carcinoma. Endoscopy 2015; 47: (Suppl. 01): E192-E194

[6] Asai S, Takeshita K, Kano Y et al. Implantation of esophageal cancer onto post-dissection ulcer after gastric endoscopic submucosal dissection. World J Gastroenterol 2016; 22: 2855 - 2860

[7] Inoue T, Fujii H, Koyama F et al. Intraluminal lavage to remove exfoliated tumor cells after colorectal endoscopic submucosal dissection. Surg Endosc 2016130: 2773-2778

[8] Tanaka S, Haruma K, Tanimoto T et al. Ki 67 and transforming growth factor alpha(TGF-a) expression in colorectal recurrent tumors after endoscopic resection. Recent Adv Gastroenterol Carcinogen 1996; 1 : $1079-1083$

[9] Rahmi G, Tanaka S, Ohara Y et al. Efficacy of endoscopic submucosal dissection for residual or recurrent superficial colorectal tumors after endoscopic mucosal resection. J Dig Dis 2015; 16: 14-21

[10] Toyonaga T, Ohara Y, Baba S et al. Peranal endoscopic myectomy (PAEM) for rectal lesions with severe fibrosis and exhibiting the muscle-retracting sign. Endoscopy 2018; 50: 813-817 